\title{
QUBIC: A Fizeau Interferometer Targeting Primordial B-Modes
}

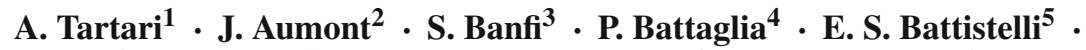
A. Baù ${ }^{3}$ - B. Bélier ${ }^{6}$ - D. Bennett ${ }^{7}$ - L. Bergé ${ }^{8}$ - J. Ph. Bernard ${ }^{9}$. M. Bersanelli ${ }^{4}$ - M. A. Bigot-Sazy ${ }^{1}$ - N. Bleurvacq ${ }^{1}$ - G. Bordier ${ }^{6}$. J. Brossard ${ }^{1}$ - E. F. Bunn ${ }^{10}$ - D. Buzi ${ }^{5}$ - D. Cammilleri ${ }^{1}$ - F. Cavaliere ${ }^{4}$. P. Chanial ${ }^{1}$ - C. Chapron ${ }^{1}$ - A. Coppolecchia ${ }^{5}$ - G. D'Alessandro ${ }^{5}$. P. De Bernardis ${ }^{5}$ - T. Decourcelle ${ }^{1}$ - F. Del Torto ${ }^{4}$ - M. De Petris ${ }^{5}$. L. Dumoulin 8 - C. Franceschet ${ }^{4}$ - A. Gault ${ }^{11}$ - D. Gayer ${ }^{7}$ - M. Gervasi ${ }^{3}$. A. Ghribi ${ }^{1}$ - M. Giard ${ }^{9}$ - Y. Giraud-Héraud ${ }^{1}$ - M. Gradziel ${ }^{7}$ - L. Grandsire ${ }^{1}$. J. Ch. Hamilton ${ }^{1}$ - V. Haynes ${ }^{12}$ - N. Holtzer ${ }^{8}$ - J. Kaplan' ${ }^{1}$ A. Korotkov ${ }^{13}$. J. Lande ${ }^{8}$ - A. Lowitz ${ }^{11}$ - B. Maffei ${ }^{12}$ - S. Marnieros ${ }^{8}$ - J. Martino' ${ }^{1}$. S. Masi ${ }^{5}$ - M. McCulloch ${ }^{12}$ - S. Melhuish ${ }^{12}$ - A. Mennella ${ }^{4}$ L. Montier9 ${ }^{9}$ A. Murphy ${ }^{7}$ - D. Néel ${ }^{8}$ - M. W. $\mathrm{Ng}^{12}$ - C. O'Sullivan ${ }^{7}$ - F. Pajot $^{2}$.

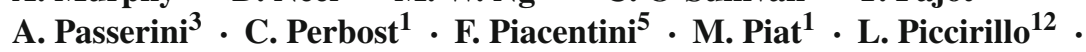

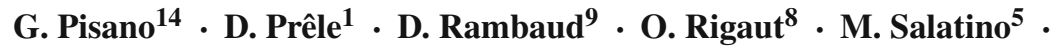
A. Schillaci ${ }^{5}$ - S. Scully ${ }^{7}$ - M. M. Stolpovskiy ${ }^{1}$ - P. Timbie T1 $^{11}$. G. Tucker $^{13}$. D. Viganò ${ }^{4}$ - F. Voisin ${ }^{1}$ - B. Watson ${ }^{12}$ - M. Zannoni ${ }^{3}$

Received: 16 September 2015 / Accepted: 30 November 2015 / Published online: 29 December 2015 (C) Springer Science+Business Media New York 2015

Abstract Q and U Bolometric Interferometer for Cosmology (QUBIC) is a Fizeau interferometer sensitive to linear polarisation, to be deployed at the Antarctic station of Dome C. This experiment in its final configuration will be operated at 97, 150

\footnotetext{
A. Tartari

tartari@apc.univ-paris7.fr; tartari@apc.in2p3.fr

1 Laboratoire APC - CNRS, Université Paris-Diderot, 75205 Paris, France

2 Institut d'Astrophysique Spatiale, Orsay, France

3 Università degli Studi di Milano-Bicocca, Milan, Italy

4 Università degli Studi di Milano, Milan, Italy

5 Università di Roma La Sapienza, Rome, Italy

6 Institut d'Electronique Fondamentale, Orsay, France

7 NUI, Maynooth, Ireland

8 CSNSM, Univ Paris-Sud, CNRS/IN2P3, 91405 Orsay, France

9 Institut de Recherche en Astrophysique et Planétologie, Toulouse, France
} 
and $220 \mathrm{GHz}$ and is intended to target $\mathrm{CMB}$ primordial B-modes in a multipole window $20<\ell<150$. A sensitivity of $r=0.05$ (95\% CL) can be reached by the first module alone, after 2 years of operation. Here we review in particular its working principles, and we show how the QUBIC interferometric configuration can be considered equivalent to a pupil-plane filtered imaging system. In this context, we show how our instrument can be self-calibrated. Finally, we conclude by showing an overview of the first dual-band module $(150 / 220 \mathrm{GHz})$, which will serve also as a demonstrator for the subsequent units, and review the technological choices we made for each subsystem, with particular emphasis on the detection system.

Keywords Cosmic microwave background $\cdot$ Polarisation $\cdot$ B-mode

\section{Introduction}

The importance of primordial B-mode detection can hardly be overestimated [1]. We know that from primordial B-mode amplitude measurements, knowing the amplitude of the stronger E-mode signal, we can infer the energy scale at which inflation occurred. In fact, the ratio of B-mode and E-mode angular power spectra at $\ell=2$ gives directly the tensor to scalar ratio $r$, while the inflation energy scale is related to $r$ through the relation $V^{1 / 4}=1.06 \times 10^{16} \mathrm{GeV}(r / 0.01)^{1 / 4}$. The Q and U Bolometric Interferometer for Cosmology (QUBIC) experiment [2], conceived to target primordial B-modes, in its full implementation consisting of six modules, will observe the sky at 97,150 and $220 \mathrm{GHz}$ from the Antarctic station of Dome C. The first module, initially intended to be operated at $150 \mathrm{GHz}$ (see, for instance, the LTD-15 paper [3]), will be instead a dual-band instrument operating at $150 / 220 \mathrm{GHz}$. This choice has been dictated by the first detection of a signal in the BB spectrum by the BICEP2 team [4], with a corresponding $r$ parameter close to 0.2 , and by its controversial interpretation. In fact, the joint BICEP2/Keck Array/Planck study [5] has revealed an unexpectedly high level of polarised dust contamination in $\mathrm{CMB}$ bands that could basically explain the overall detected B-mode signal. In this context, we decided to modify the architecture of the first module in order to add a dust channel $(220 \mathrm{GHz})$, at the price of a slightly reduced sensitivity at $150 \mathrm{GHz}$. Here we recall the principles of operation of the instrument, we describe the progress in the realisation of the first module (underlining the impact of the recent dual-band modification), and we review some of the critical subsystems.

\footnotetext{
10 University of Richmond, Richmond VA, USA

11 University of Wisconsin, Madison, WI, USA

12 School of Physics and Astronomy, University of Manchester, Manchester, UK

13 Brown University, Providence, RI, USA

14 School of Physics and Astronomy, University of Cardiff, Cardiff, UK
} 

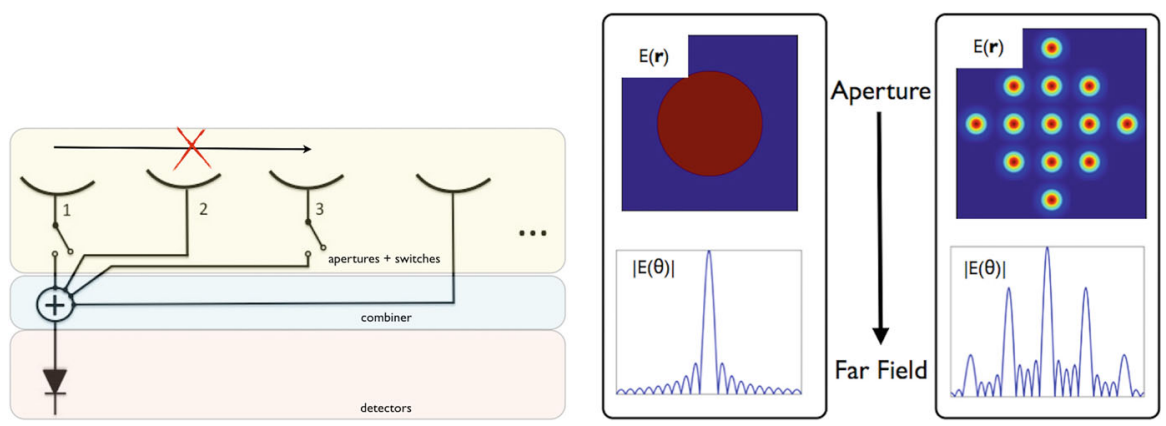

Fig. 1 Left The self-calibration configuration: a pair of horns (1 baseline corresponding to the specific realisation of a Fourier mode) can be excluded by means of waveguide switches; Right QUBIC can be considered as an imager whose pupil, instead of being uniformly illuminated, is sampled by a series of apertures each one with a Gaussian field profile (Color figure online)

\section{QUBIC as an Adding Interferometer}

The most common technique to make mm-wave interferometers is certainly multiplicative (heterodyne) interferometry. In this case, long baselines (up to $10 \mathrm{~km}$, or more) can be achieved, and IF bandwidth $\sim$ some GHz wide allows sensitive operation when observing continuum sources. The drawback is that pairwise correlation of antennas is achieved through fast digital correlators, whose complexity and costs make it practically unfeasible for systems with hundreds of antennas. In adding interferometry, on the contrary, correlation is achieved through linear combination of fields followed by direct detection, so that hundreds of signals can be correlated at a time, provided a suitable adding scheme is envisaged. In the particular case of an adding all-to-one interferometer, all the signals are combined on each detector (see Fig. 1, left panel), so that each detector's output is a linear combination of total intensities collected by each antenna, plus interference terms that are proportional to the visibilities corresponding to the Fourier modes selected by the horn antenna array. In the case of QUBIC, the sky is observed through 400 closely packed back-to-back horns: the sky horn beam defines the field of view of the instrument, while the back horns illuminate a fast telescope, acting as a beam combiner. In fact, families of parallel rays re-emitted from the back horns are summed in phase on each detector of a pair of kilo-pixel arrays. This is a specific implementation of a general Fizeau interferometer scheme. The system of back-to-back horns can be regarded also as a spatial filter in front of a telescope (our combiner), selecting the Fourier modes accepted by our system pupil. According to this view, the synthetic beam through which each pixel observes the sky is the Fourier transform of the electric field distribution at the level of sky horn apertures (see Fig. 1, right panel, for a toy model representation of the concept). The synthetic beam, unlike the one arising from a uniformly illuminated aperture, shows higher-order peaks (exactly like in the schematic representation of Fig. 1) that must be used for image reconstruction together with the main beam. In the practical case of QUBIC, the number of secondary peaks to be used is of the order of 10 . What we have described up to now is a telescope exploiting a subset of Fourier modes (among those 
that would be otherwise collected by the telescope without the horn array in front of it) to produce an image. There would be no point in the choice of this architecture if it was not for the presence of a waveguide switch in each back-to-back (see Fig. 1, left panel): thanks to this feature, a given baseline can be characterised independently, and a set of baselines realising the same Fourier mode (equivalent baselines) can be used to obtain a largely redundant number of measurements of an artificial far-field source to recover the systematics of the instrument, encoded in a certain number of unknown parameters describing horn cross-polarisation, transmission unbalancing, etc. (see [6] for a comprehensive discussion). In this way, the instrument can be self-calibrated, and a novel way of handling systematics can be exploited.

\section{The First Module}

The design of the new module has been modified to cover 150 and $220 \mathrm{GHz}$ channels instead of just the $150 \mathrm{GHz}$ band for foreground subtraction purposes. The main hardware modifications are the following: (1) the polarising grid that was originally placed after the beam combiner to split the two orthogonal polarisation components on two different focal planes is now put in front of the feedhorn array; (2) the grid is replaced by a dichroic in order to separate the two frequency bands (instead of the two polarisations). The main drawback of this choice is that we loose half of the signal at $150 \mathrm{GHz}$ (reflected towards the sky), although we gain the capability of monitoring the dust with our own data set. All the other components are basically unchanged, although some of them will be slightly modified to accommodate the higher frequency channel. In the following, we describe the status of the different subsystems, underlining dual-band modifications whenever this is the case.

\subsection{Cryostat and Cryogenics}

The design of the cryostat is currently being finalised. The pulse tube cryostat, with a $45 \mathrm{~cm}$ clear aperture, will accommodate the cold optics and detector box visible in Fig. 2 and will provide the $4 \mathrm{~K}$ stage to cycle a He- 4 fridge and an He-10 fridge. The $\mathrm{He} 4$ fridge will cool down to $1 \mathrm{~K}$ the shield, the cold stop, the primary and secondary mirrors, the dichroic filter and part of the readout electronics (the SQUIDs, as in Figs. 2, 3). The He-10 fridge will cool down the two TES arrays at a temperature of 320/350 mK. The half-wave plate (HWP) and the polarising grid, not shown in Fig. 2, will be at $4 \mathrm{~K}$ together with the horn array.

\subsection{Horns and Optics}

In the optical chain of the first module, completely contained within the cryostat, we find (in order from the hottest to the coldest part) an HWP, a polarising grid, the back-toback horns, the beam combiner and a dichroic filter. The HWP, based on dielectrically embedded mesh technology [7], is designed to have an achromatic behaviour across the two QUBIC bands, where its transmission is expected to be $>90 \%$. The corrugated 


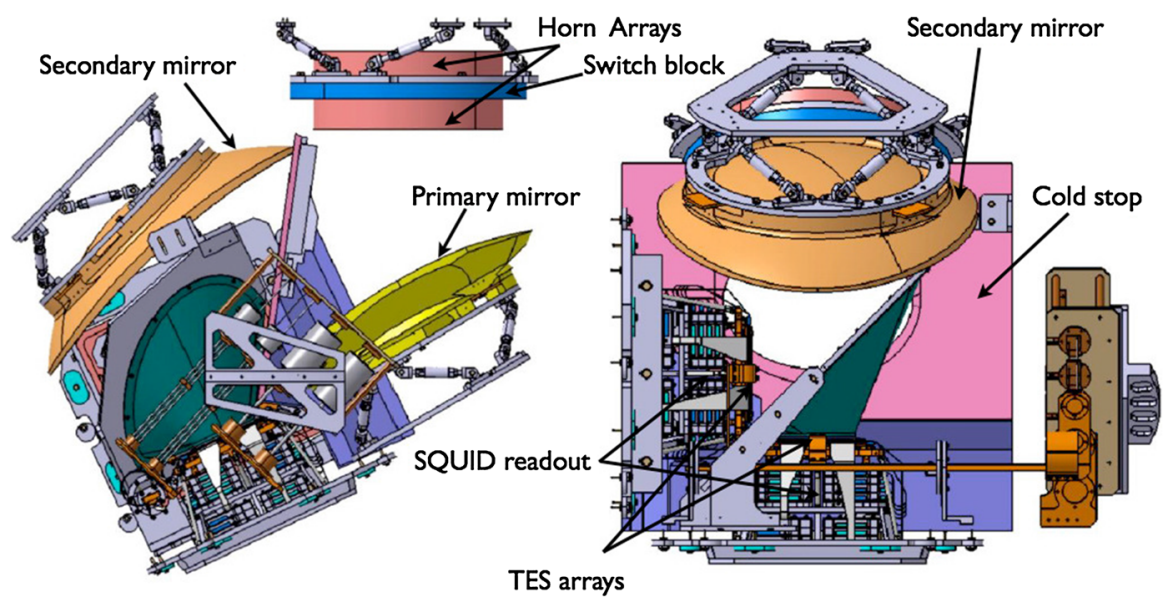

Fig. 2 Two views of the cold optics and detector box. In evidence also the back-to-back and switch block, suspended on the telescope (beam combiner) (Color figure online)

horns are the only part of the instrument redesigned in view of dual-band operation, basically to extend to $220 \mathrm{GHz}$ the good properties already demonstrated at $150 \mathrm{GHz}$. In particular, with the new design the return loss of the fundamental mode is now <$25 \mathrm{~dB}$ from 140 to $230 \mathrm{GHz}$. Manufacturing of $8 \times 8$ prototypes in platelet technology is in progress and will allow to assess the RF performance of our back-to-back, including crosstalk.

\subsection{Detectors and Detector Readout Electronics}

QUBIC detectors are NbSi TES sensors, with a critical temperature tuned at $400 \mathrm{mK}$ for the first module. They are arranged in a filled array configuration, suitable for the imaging of interference fringes with an high filling factor. Light is coupled to the detectors through $\mathrm{TiV}$ free-space matched absorbers, suspended on a SiN membrane along with the NbSi thermistors. As usual, absorption is maximised by means of a metallic $\lambda / 4$ backshort. Each of the two kilo-pixel arrays consist of four tiles of 248 detectors each (see Fig. 3, left panel). QUBIC array fabrication, described in [8], is currently progress. Concerning the readout, QUBIC exploits a state-of-the-art 128:1 time-domain multiplexing electronics [9], featuring a SiGe ASIC operating at 40K per 128 SQUIDs (at 1K). Preliminary tests have been performed on the very first sample available, with the complete readout chain operational, and the results are encouraging in terms of homogeneity (see Fig. 3, right panel), despite a yield lower than expected obtained for this specific sample. From electrical measurements alone (see the $\mathrm{P}-\mathrm{V}$ and $\mathrm{I}-\mathrm{V}$ curves in Fig. 3, where the action of electrothermal feedback is clearly displayed), we could infer a thermal conductance of the order $G \sim 84 \mathrm{pW} / \mathrm{K}$, corresponding to $\mathrm{NEP}_{\text {phonon }} \sim 3 \times 10^{-17} \mathrm{~W} / \sqrt{\mathrm{Hz}}$, a value which matches QUBIC specifications. The reliability of all the fabrication processes is being assessed in view of the production of the arrays to be installed in the focal plane of QUBIC. 


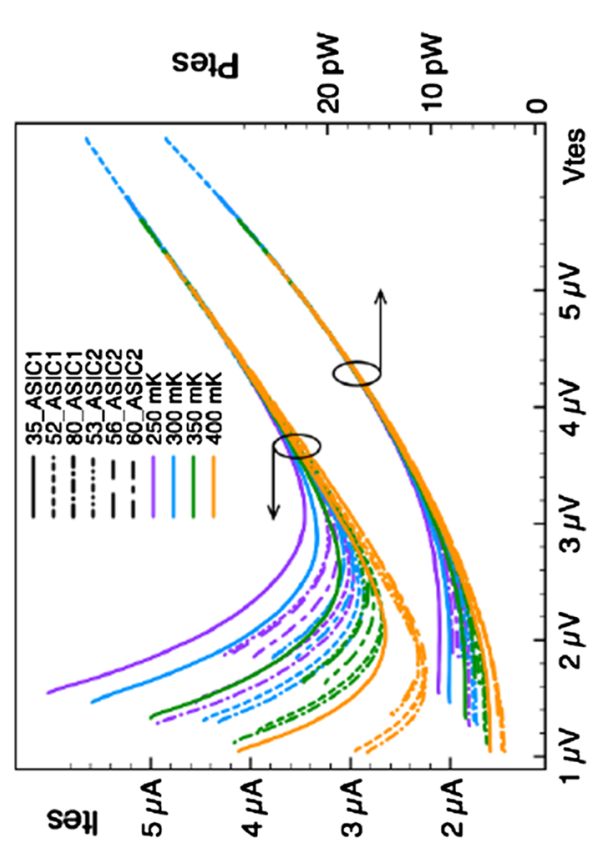

를

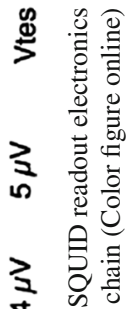

o

$>\quad \frac{0}{0}$

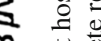

吾 $\frac{0}{2}$

艺

包

를

苋

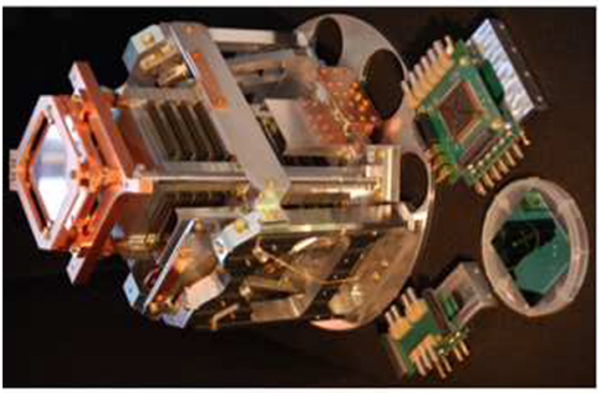

กุ่

르륰

$\cong$ 표

플

吾放

赵离

is 요

는

$\overline{0}$

후원

능

흐

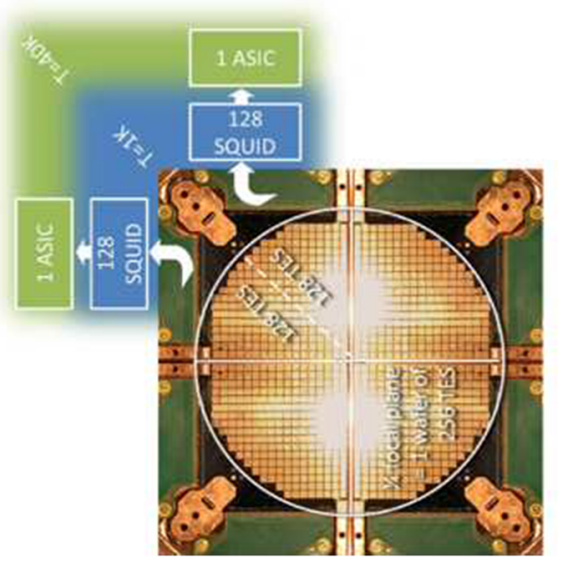

3

$\stackrel{\Xi}{\Xi}$

1

등

$\mathbb{D}^{\circ}>$

핌

ㅊ. 옹

ic

式

के

है

งั

n $\frac{\pi}{2}$

艎 


\section{Conclusions and Perspectives}

The first module of QUBIC will be implemented in a dual-band configuration adequate to reach ambitious science goals by itself and suitable to demonstrate the validity of the instrument concept in view of the deployment of the five subsequent modules. The first module alone, observing the sky for 2 years (with a realistic time efficiency of $30 \%$, also considering repeated sequences of self-calibration), will be able to set $r<0.05$ at $95 \% \mathrm{CL}$ in the presence of galactic dust. This experiment has novelty elements in the way of dealing with systematics and for this very reason can play an important role among the experiments searching for primordial B-modes. The design of all the subsystems is currently frozen, and no major issues are expected with the current design. The integration of the first module will take place in the second half of 2016.

Acknowledgments We acknowledge the financial support from UnivEarthS Labex programme of Sorbonne Paris Cité (ANR-10-LABX-0023 and ANR-11-IDEX-0005-02), the CNRS, the ANR and the Region Ile de France that is supporting us through the DIM-ACAV programme. We acknowledge also the financial support from PNRA.

\section{References}

1. D. Baumann, et al. arXiv:0811.3919v2 (2009)

2. The QUBIC Collaboration, Astropart. Phys. 34, 705-716 (2011)

3. A. Ghribi et al., J. Low Temp. Phys. 176, 698-704 (2014)

4. The BICEP2 Collaboration, PRL 112, 241101 (2014)

5. BICEP2/Keck/Planck Collaborations, PRL 114, 101301 (2015)

6. M.-A. Bigot-Sazy, A\&A 550, 59B (2013)

7. G. Pisano, M.W. Ng, V. Haynes, B. Maffei, Prog. Electromagn. Res. M 25, 101-114 (2012)

8. C. Perbost, et al., A 248 TES Array for the detection of CMB B-mode polarization. J. Low Temp. Phys. (2015, submitted)

9. D. Prêle, et al., A 128 multiplexing rate time domain SQUID multiplexer. J. Low Temp. Phys. (2015, submitted) 